\title{
A PRELOAD AND AFTERLOAD SENSITIVE ARTIFICIAL VENTRICLE TO TEST CARDIOVASCULAR PROSTHESES
}

\author{
Maurizio Arabia* Guido Danieli * \\ Francesco Maria Colacino* Francesco Moscato* \\ Salvatore Nicosia ${ }^{* *}$ Fabio Piedimonte ${ }^{* *}$ \\ * Dip. di Meccanica, Università della Calabria, \\ Arcavacata di Rende, CS, Italia \\ ** Dip. Informatica, Sistemi, Produzione; Università di \\ Roma TorVergata, Roma, Italia \\ piedimonte@robot2.disp. uniroma2. it
}

\begin{abstract}
In the last decades cardiovascular diseases greatly increased worldwide, and bioengineering provided new technologies and cardiovascular prostheses to medical doctors and surgeons. Ventricular assist devices aroused notable interests. As a consequence it is important to faithfully reproduce the interaction between the prostheses and the cardiovascular system when in-vitro experiments are performed. For this reason, a new and improved kind of test benches become necessary. In this paper an artificial ventricle connected to a hydraulic circuit is described. The ventricle's control architecture is based on the estimation of some characteristic parameters. It is showed how this control strategy leads to the mutual interaction between the artificial ventricle and the hydraulic circuit and a correct mean atrial (preload) and aortic (afterload) pressure sensitivity and hydrodynamics. Copyright (c) 2005 IFAC
\end{abstract}

Keywords: Cardiovascular prostheses, ventricular assist devices (VADs), artificial ventricle, time varying elastance model, parameter estimation.

\section{INTRODUCTION, AIM AND METHODS}

Hydraulic mock circulatory systems (MCSs) are widely used to test mechanical cardiac assist devices (CADs) (Baloa et al., 2001), (Pantalos et al., 2004), (Shi et al., 2004) and (Matthew and Yih-Choung, 2004). They are an important tool for CADs' design and testing. On the other hand, reproducibility of inlet and outlet pressures and/or flow conditions in CADs, by means of hydraulic components, is a complex and challenging issue. Hydraulic feasibility of complex mathe-

\footnotetext{
1 Partially supported by the Italian Ministry of Research
} - FIRB RBAU0123CR matical models describing the phenomenon under study, low flexibility when different devices must be tested or significant parameters changed in real time, and the total cost of hydraulic components with respect to those numerically simulated are the main limits of hydraulic MCSs. Also, every hydraulic component is always affected by spurious effects (i.e. a hydraulic resistance will always be coupled to a small inertance). Due to these limitations and by considering how flexible, precise, accurate and costless mathematical models are if compared with their analog hydraulic components, a new test bench (TB) must be conceived to conjugate numerical simulations with the hy- 
draulic environment the cardiovascular prostheses are supposed to operate in.

Aim of this paper is to describe an artificial ventricle sensitive to variations of mean atrial (preload) and aortic (afterload) pressures suitable for testing CADs. In particular, the correct design of VADs plays a key role in cardiovascular bioengineering. VADs were originally developed as bridges to transplant, but if properly controlled and under particular conditions, show the capability to recover pathologic hearts. Although it is not the optimal solution yet, the proposed system is an important improvement of present test benches towards a correct development of these prostheses.

In order to achieve this result, the control system of the artificial ventricle must be sensitive to different working conditions the prosthesis is underwent to. Hence, such a ventricle must be controlled in order to respond to variations which take place into the cardiovascular system. In particular, the instantaneous ventricular pressure must be determined on the basis of the instantaneous ventricular volume. This control strategy is based on a pressure controller under development and test (Arabia et al., 2004). The main issue is its robustness. The working conditions the pressure controller must respond to are characterized by strong parametric variations and involve different physical components which can be also different if the experimental setup changes.

Instead of using the instantaneous ventricular pressure as control variable, the control strategy proposed in this paper is based on the mean ventricular pressure. This is calculated in one cardiac cycle and in correspondence of some points of the hydraulic circuit. This result is obtained by choosing a real-time simulated flow rate waveform in one cardiac cycle as input to the system. Some parameters of this simulation are tuned to properly estimate some physical variables of the hydraulic circuit.

The paper is organized as follows: in section 2, models of the cardiovascular system are introduced; in section 3, the experimental apparatus used is described; in section 4 , the attention is focused on the control architecture of the test bench; in section 5, experimental results are showed and discussed.

\section{CARDIOVASCULAR MODELS}

The cardiovascular system (Guyton, 1986) comprises ventricles, atria, valves, and systemic and pulmonary circulations. A widely used approximation is to consider only the systemic circulation and the left ventricle preceded by the left atrium (Guyton et al., 1973).

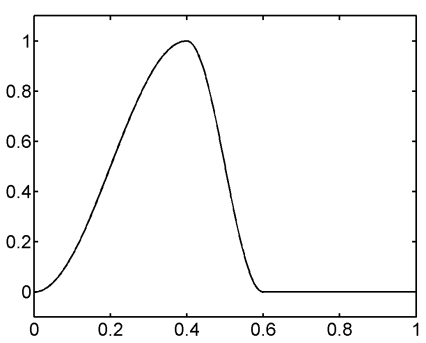

Fig. 1. Ventricular contraction function Fiso

The ventricle is modeled by the time varying differential elastance model with internal resistance (Arabia et al., 2003), (Vollkron et al., 2002) and (Campbell et al., 1984). This model links the instantaneous ventricular pressure with the instantaneous ventricular volume in one cardiac cycle. In a simplified form this relation is:

$$
P_{l v}(t)=P_{0}+\varphi\left[V_{l v}(t), t\right]-R \frac{d V_{l v}(t)}{d t}
$$

where $V_{l v}(t)=$ instantaneous blood volume inside the ventricle, $P_{l v}(t)=$ instantaneous blood pressure inside the ventricle, $R=$ internal resistance (it represents the internal dissipation of energy during the ejection phase). The fixed point having coordinates $\left(V_{0}, P_{0}\right)$ is the pivot point of the function $\varphi\left[V_{l v}(t), t\right]$ for variations of $V_{l v}(t)$ and $t$. At a generic instant $t$, the differential elastance is the slope of the tangent to the function $\varphi\left[V_{l v}(t), t\right]$ at a generic volume $V_{l v}(t)$. The function $\varphi\left[V_{l v}(t), t\right]$ is as follows:

$$
\begin{aligned}
\varphi\left[V_{l v}(t), t\right] & =\varphi_{p}\left[V_{l v}(t)\right]+ \\
& +\left(\varphi_{a}\left[V_{l v}(t)\right]-\varphi_{p}\left[V_{l v}(t)\right]\right) F_{i s o}(\theta)(2)
\end{aligned}
$$

where $\varphi_{p}$ and $\varphi_{a}$ represent the elastance curves when the ventricle is at rest (passive), and maximally stimulated (active), respectively; $\theta=\frac{t}{T}$, $T$ is the cardiac period; $F_{i s o}(\theta)$ (fig. 1 ) is the normalized contraction function, which is invariant with respect to different working conditions. The function $F_{i s o}(\theta)$ recurs every cardiac cycle. It is obtained by means of two sinusoids and an interval during which is equal to zero.

The function $\varphi_{p}$, named end-diastolic pressurevolume relationship (EDPVR), has been modeled by the sum of a straight line and an equilateral hyperbola, whose vertical asymptote represents the filling saturation volume of the ventricle. $\varphi_{a}$, named end-systolic pressure-volume relationship (ESPVR), has been modeled by a parabola with downward concavity.

Atrial contractility has been ignored and a capacitor has been used to model the atrium. The venous return comprises one resistance and one capacitor according to the Guyton model (Guyton et al., 1973); the arterial load has been modeled by 


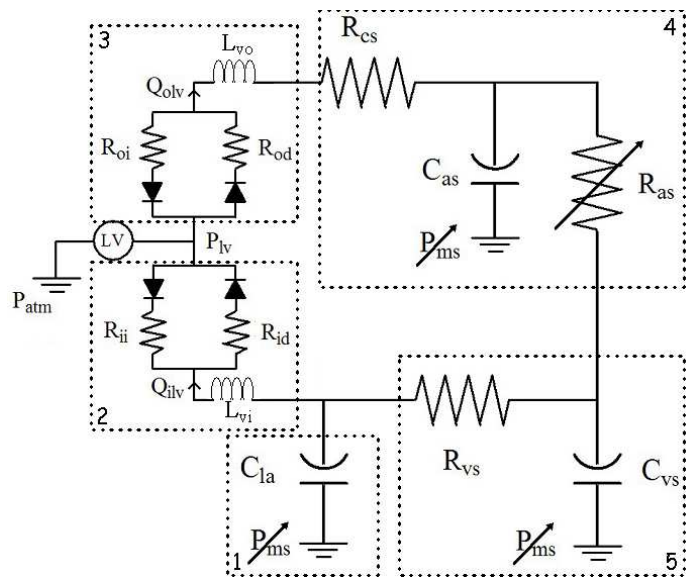

Fig. 2. 1: atrium, 2: inlet valve, 3 : outlet valve, 4: arterial systemic load - Modified Windkessel model, 5: venous return - Guyton's model, LV: left ventricle

a three-component Windkessel model, comprising two resistances and one capacitor (Westerhof et al., 1971). Inlet and outlet valves are modeled as resistances, whose values are different in case of forward and backward flow, and are in series connection with an inductor. Figure 2 shows the electric analog of this cardiovascular system.

A powerful tool for the analysis of the mutual interactions between heart and vascular system is the ventricular pressure-volume (PV) loop. It is important to analyze how the PV loop changes for variations of preload and afterload (Sagawa et al., 1988), or due to aging and pathological conditions. In details, a preload increase leads to an increase of both mean ventricular volume and stroke volume, while an afterload increase leads to an increase of mean ventricular volume and a decrease of stroke volume. This behavior is known as Starling law (Starling, 1918). All the pathologies cause deformations of the PV loop. Therefore, one of the aims of the test bench is to verify how the PV loop changes when preload and afterload change due to the action of an implanted VAD. This is useful to judge the efficacy of a VAD as a therapy. Also, a test bench is useful to test the hemodynamics and the structural behavior of the VAD itself.

\section{HYDRAULIC LAYOUT}

A piston-cylinder mechanism has been designed and manufactured to be used as a ventricle. It is able to provide a stroke volume up to 200 $[\mathrm{ml}]$. This allows covering the widest range of working conditions corresponding to different endsystolic and end-diastolic volumes. The driving system is made up of a screw mechanism. The DC motor (Maxon RE 35) is servo controlled by a 4 quadrant driver (Maxon ADS 50/5). The motor

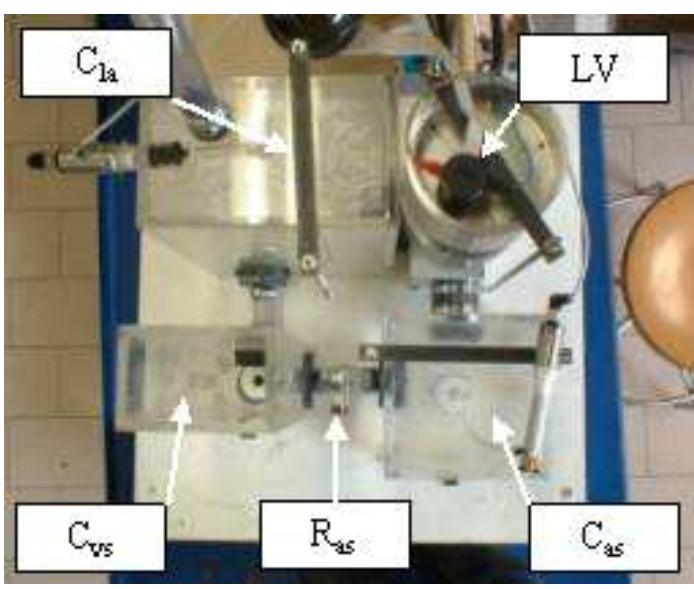

Fig. 3. Hydraulic layout

speed is the control variable and is monitored by a built-in encoder.

The hydraulic afterload and venous return have been reproduced by modified Windkessel and Guyton models, respectively (fig. 3). This closed loop setup represents a hydraulic simulator of the systemic circulation with left atrium. Capacitors are realized by means of reservoirs, while resistances and inductors by rigid tubes (no compliant effects). At the inlet and outlet of the artificial ventricle two artificial valves have been placed.

Three pressure transducers (Tekkal) are used to measure pressure into the ventricular chamber, as well as into the arterial systemic and atrial compliances.

The DAQ board is a Sensoray (mod. 626). The real time application is implemented by means of RTLinux which allows efficient management of input and output signals. Every real time process is directly controlled by RTLinux without involving the user.

\section{CONTROL STRATEGY}

The control strategy chosen is based on the following idea: if the model of the system is highly accurate, then it will be possible to use the flow rate waveforms as input signal of an open loop system, and get the correct pressure waveforms as output. In order to achieve this result, all the components of the loop have been characterized, including those spurious always present in physical systems. Nevertheless, the evaluation of some parameters of the hydraulic circuit will not be feasible, if the rig will not be running. For example, the mean systemic pressure $P_{m s}$ is increased or decreased by respectively introducing into or depleting from the reservoir named $C_{v s}$ water when preload variations must be obtained. In a similar way, the arterial systemic resistance $R_{a s}$ is increased by occluding the arterial out- 


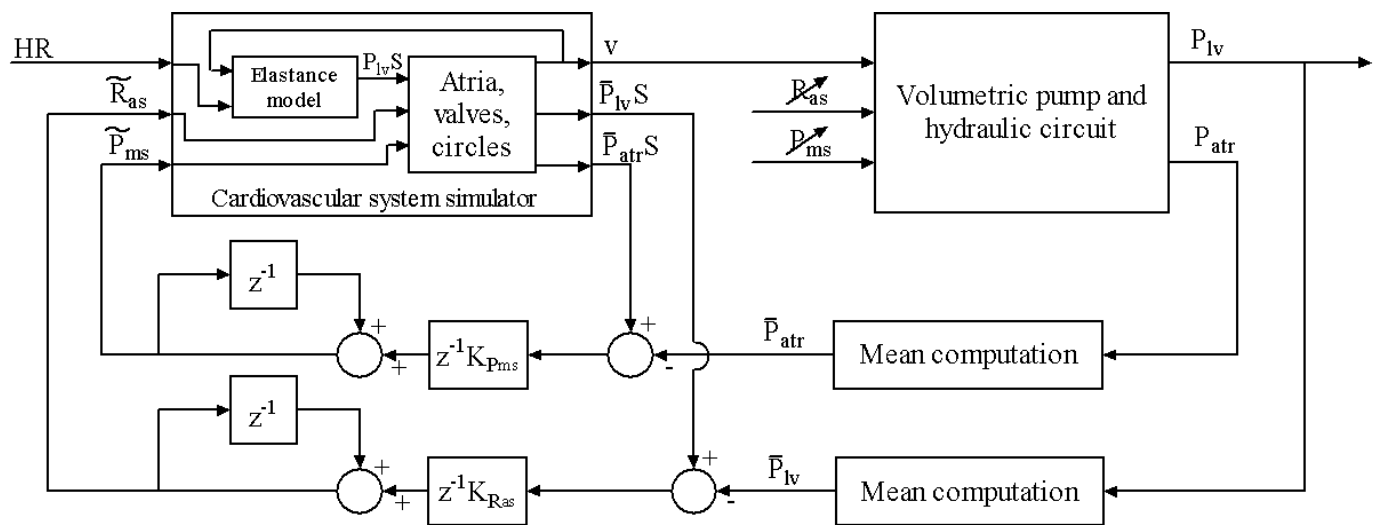

Fig. 4. Control strategy scheme. $H R$ : heart rate; $v$ : motor speed; $P_{a t r}$ : atrial pressure; ${ }^{\sim}$ is for estimated quantities.

flow with a turn style resistor clamp. Whereas preload is almost independent from the arterial systemic resistance $R_{a s}$, afterload is not totally independent from $P_{m s}$ variations. Since these two parameters are not known a-priori, it becomes necessary to continuously estimate them while the experiment is running. The real time variations of $P_{m s}$ and $R_{a s}$ in the simulated model lead to the correct sensitivity of the test bench to preload and afterload changes.

In particular, a $P_{m s}$ increase leads to an increase of all the mean pressures (atrial, ventricular and aortic) into the hydraulic circuit; while if $R_{a s}$ increases, the mean aortic and ventricular pressures will increase, but variations of the mean atrial pressure will be negligible. Therefore, to estimate $R_{a s}$ and $P_{m s}$ the mean ventricular pressure and the mean atrial pressure are chosen, respectively.

Figure 4 shows the scheme of the control strategy adopted: a real time simulation of the bench starts, its output is the reference signal for the pump motor speed (an instantaneous and accurate servo-controller speed is supposed). The unknown parameters are continuously estimated by means of the difference between simulated and measured mean pressures. In particular, if the measured mean atrial pressure $\left(\bar{P}_{a t r}\right)$ is smaller than the simulated one $\left(\bar{P}_{a t r} S\right)$, then it means that the current value of $P_{m s}$ into the model is too high and, as a consequence, will be diminished by an amount proportional to the difference between the two mean pressures. Mathematically $\left(K_{P_{m s}}\right.$ is a gain):

$$
\begin{aligned}
P_{m s}(k+1) & =P_{m s}(k)+ \\
& +K_{P_{m s}}\left[\bar{P}_{a t r}(k)-\bar{P}_{a t r} S(k)\right]
\end{aligned}
$$

The estimate of $R_{a s}$ is performed in a similar way: if the measured mean ventricular pressure is smaller than the simulated one, then it means that the current value of $R_{a s}$ is too high and, as a consequence, will be diminished by an amount proportional to the difference between the two mean pressures. Mathematically ( $K_{R_{a s}}$ is a gain):

$$
\begin{aligned}
\widetilde{R}_{a s}(k+1) & =R_{a s}(k)+ \\
& +K_{R_{a s}}\left[\bar{P}_{l v}(k)-\bar{P}_{l v}(k) S\right]
\end{aligned}
$$

The mean values are calculated in one cardiac cycle, either at the end or during the cardiac cycle itself by using the differential average method. In the first case, the difference between simulated and measured pressure signals is maintained constant during the entire cycle, while in the second case this difference varies between two sampling instants. The first method is slower but easier to implement.

The greater the gains $K_{P_{m s}}$ and $K_{R_{a s}}$, the faster is the estimate, but for too high values the system becomes unstable. Under the hypothesis of step variations of $P_{m s}$ and $R_{a s}$, after a transient the mean pressures converge and the estimation is correct.

The idea that leads the estimated parameters to converge to real values and the mean pressures to real signals follows. The transfer matrices below are in $\mathcal{Z}$-Domain. The transfer matrix of the block in figure 4 named Volumetric pump and hydraulic circuit is assumed to be as follows:

$$
\left[\begin{array}{c}
P_{l v} \\
\bar{P}_{a t r} \\
\bar{P}_{l v}
\end{array}\right]=\left[\begin{array}{ccc}
P_{11} & P_{12} & P_{13} \\
P_{21} & P_{22} & 0 \\
P_{31} & P_{32} & P_{33}
\end{array}\right]\left[\begin{array}{c}
v \\
P_{m s} \\
R_{a s}
\end{array}\right]
$$

where $P_{23}=0$ under the hypothesis that the mean atrial pressure is independent from afterload variations. The block named Atria, valves, circles has the following form:

$$
\left[\begin{array}{c}
v \\
\bar{P}_{a t r} S \\
\bar{P}_{l v} S
\end{array}\right]=\mathcal{P}\left[\begin{array}{c}
P_{l v} S \\
\widetilde{P}_{m s} \\
\widetilde{R}_{a s}
\end{array}\right]
$$




$$
\mathcal{P}=\left[\begin{array}{ccc}
\frac{1}{P_{11}} & -\frac{P_{12}}{P_{11}} & -\frac{P_{13}}{P_{11}} \\
\frac{P_{21}}{P_{11}} P_{22}-\frac{P_{21} P_{12}}{P_{11}} & -\frac{P_{21} P_{13}}{P_{11}} \\
\frac{P_{31}}{P_{11}} & P_{32}-\frac{P_{31} P_{12}}{P_{11}} & P_{33}-\frac{P_{31} P_{13}}{P_{11}}
\end{array}\right]
$$

Matrix in 6 is obtained from 5 under the assumption that the equations of the physical components of the bench are well known. Also, if $\bar{P}_{a t r} S=$ $\bar{P}_{a t r}, \bar{P}_{l v} S=\bar{P}_{l v}, \widetilde{P}_{m s}=P_{m s}, \widetilde{R}_{a s}=R_{a s}$ and $P_{l v} S=P_{l v}$ and 6 is substituted into 5 , the result is the identity matrix. Moreover systems 5 and 6 must be stable. By correspondently substituting rows in 5 and 6 into 3 and 4 in $\mathcal{Z}$-domain, the following relationships are obtained:

$$
\begin{aligned}
z \widetilde{P}_{m s}(z) & =\widetilde{P}_{m s}(z)+ \\
& +K_{P_{m s}}\left[P_{22}(z)\left(P_{m s}(z)-\widetilde{P}_{m s}(z)\right)\right](7) \\
z \widetilde{R}_{a s}(z) & =\widetilde{R}_{a s}(z)+ \\
& +K_{R_{m s}}\left[P_{32}(z)\left(P_{m s}(z)-\widetilde{P}_{m s}(z)\right)+\right. \\
& \left.+P_{33}(z)\left(R_{a s}(z)-\widetilde{R}_{a s}(z)\right)\right]
\end{aligned}
$$

From 7 it follows:

$$
\widetilde{P}_{m s}(z)=\frac{K_{P_{m s}} n_{P_{22}}(z) P_{m s}(z)}{(z-1) d_{P_{22}}(z)+K_{P_{m s}} n_{P_{22}}(z)}
$$

where $n_{P_{22}}$ and $d_{P_{22}}$ are numerator and denominator of $P_{22}$. By applying the final value theorem for $\mathcal{Z}$-transforms to 9 , by assuming a step as input, if $n_{P_{22}}$ does not have roots equal to 1 , and a value for $K_{P_{m s}}$ which stabilizes the system has been found, then $P_{m s}$ will be the asymptotic value of $\widetilde{P}_{m s}$. In a similar way, since $\widetilde{P}_{m s}$ converges, by substituting 9 into 8 , it is easy to show that $R_{a s}$ is the asymptotic value of $\widetilde{R}_{a s}$. Hence, from 3 and $4 \bar{P}_{a t r}$ and $\bar{P}_{l v}$ are the asymptotic values of $\bar{P}_{a t r} S$ and $\bar{P}_{l v} S$, respectively.

Note 1. The de-coupling hypothesis is heavily exploited: if it had not been possible to independently calculate at least one of the two variables, the final value theorem would not have led the parameters to converge.

Note 2. The real system is not linear and $R_{a s}$ is not properly an input, even if it is possible to consider as if it is so. Similarly, systems in 5 and 6 can be considered as a linearization of the original system. Anyway, due to the open loop configuration, the correctness of the model of the system with respect to the physical setup represents the strongest hypothesis. If differences existed, they would influence the estimate of $P_{m s}$ and $R_{a s}$. As a consequence, their values would include eventual discrepancies between real and modeled systems.
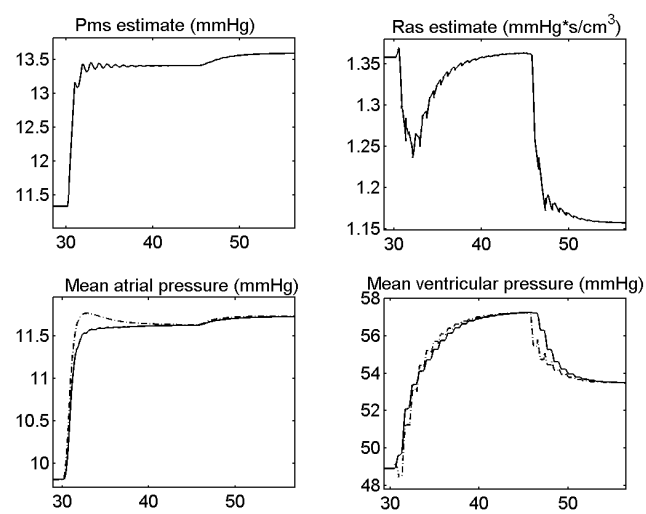

Fig. 5. Estimates (upper row); mean pressures (lower row). $\mathrm{x}$-axis time (sec)
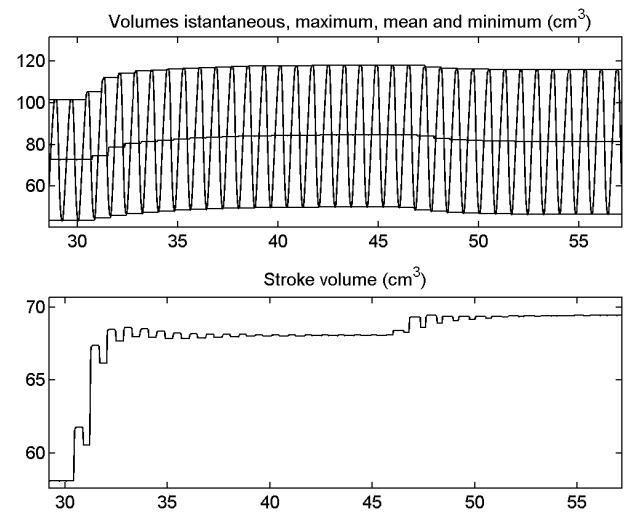

Fig. 6. Volumes. x-axis time (sec)

\section{EXPERIMENTAL RESULTS AND DISCUSSION}

This section contains the experimental results of a performed experiment. After 30 seconds from the beginning of this experiment, water is added into the hydraulic circuit to increase $P_{m s}$. Also, after other 15 seconds, $R_{a s}$ is diminished by loosening the occlusion due to the turn style resistor clamp. The upper row of Figure 5 shows the behaviour of the estimates of $P_{m s}$ and $R_{a s}$, while the lower row on the same figure shows simulated (dashed line) and experimental (thick line) mean pressures. Figure 6 shows the correct sensitivity of the test bench to preload and afterload variations: by increasing the preload, which is equivalent to $P_{m s}$ increase, the mean ventricular volume and the stroke volume increase; by decreasing the afterload, which is equivalent to $R_{a s}$ decrease, the mean ventricular volume decreases while the stroke volume increases according to the Starling's law. The pressure-volume loops are showed in figure 7 . The effects due to an increase of $P_{m s}$ are showed on the left, while on the right the effects due to a decrease of $R_{a s}$ are showed. Dashed and thick lines represent simulated and experimental data, respectively. 

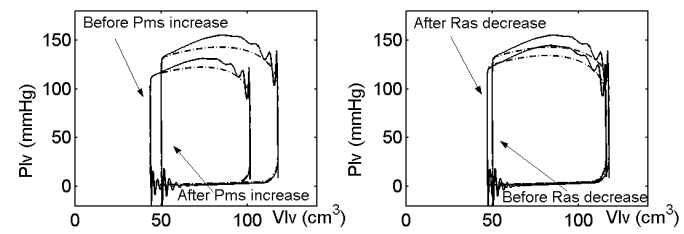

Fig. 7. Pressure-volume loops
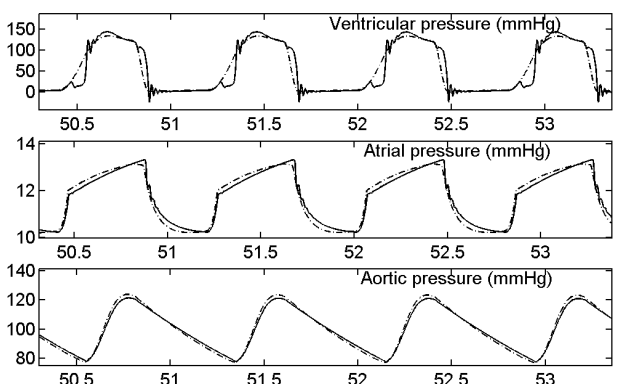

Fig. 8. Istantaneous pressures. x-axis time (sec)

One application of a preload and afterload sensitive artificial ventricle can be VAD testing. When a VAD is connected to the ventricle, the latter experiences an afterload decrease equivalent to variations of $R_{a s}$. This effect diminishes the working load of the pathological ventricle, while the patient is provided with a sufficient level of perfusion. This approximation is not far from reality.

\section{CONCLUSIONS}

Tests have been carried out and the interaction between the physical system and mathematical models has been successfully achieved. The correct sensitivity to preload and afterload variations is observed by means of a proper control strategy of the artificial ventricle.

The results obtained so far represent an intermediate step toward the design of an elastancebased artificial ventricle working as a natural ventricle while connected to the cardiovascular system and prostheses. In order to completely achieve this final task, an instantaneous ventricular pressure controller is under development. Even though mean pressure tracking works correctly, the ventricular pressure waveform is obtained by using an open loop configuration. The limitations of this control strategy are showed in figure 8 , where the instantaneous measured ventricular pressure (thick line) is deformed with respect to the simulated waveform (dashed line). Anyway, experimental atrial and aortic pressure waveforms reproduce quite faithfully those simulated.

\section{REFERENCES}

Arabia, F.M. Colacino, D. Lavorato, A.G.M. Marullo and F. Piedimonte (2003). A study on LV regional dyssynchrony based on ventricular time varying elastance computer model. In: International Congress on Computational Bioengineering. Vol. 1. Copy Center. Zaragoza. pp. 71-78.

Arabia, F.M. Colacino, G.A. Danieli, F. Moscato, S. Nicosia, S. Pagnottelli, F. Piedimonte and P. Valigi (2004). Hybrid test bench for evaluation of any device related to mechanical cardiac assistance. In: Int. J. of Art. Org.. Vol. 27(7). Wichtig. Warsaw. p. 627.

Baloa, J.R. Boston and J.F. Antaki (2001). Elastance-based control of a mock circulatory system. Annals of Biomedical Engineering 29(3), 244-251.

Campbell, J.A. Ringo, C. Neti and J.E. Alexander (1984). Informational analysis of leftventricle/systemic-arterial interaction. $A n$ nals of Biomedical Engineering 12(2), 209231.

Guyton (1986). Textbook of medical physiology. W.B. Saunders. Philadelphia.

Guyton, C.E. Jones and T.G. Coleman (1973). Circulatory physiology: cardiac output and its regulation. W.B. Saunders. Philadelphia.

Matthew and Yu Yih-Choung (2004). Feedback control design of an elastance-based mock circulatory system for heart assist device controller development. In: American control conference. The printing house. Boston.

Pantalos, M. George, Koenig, C. Steven, Gillars, J. Kevin, Giridharan, A. Guruprasad, Ewert and L. Dan (2004). Characterization of an adult mock circulation for testing cardiac support devices. ASAIO Journal 50, 37-46.

Sagawa, W.L. Maughan and K. Sunagawa H. Suga (1988). Cardiac contraction and the pressurevolume relationship. Oxford University Press. New York.

Shi, TJ Yeo and Y. Zhao (2004). Numerical simulation of a systemic flow test rig. ASAIO Journal 50, 54-64.

Starling (1918). The linacre lecture on the law of the heart. Longmans. London.

Vollkron, H. Schima L. Huber and G. Wieselthaler (2002). Interaction of the cardiovascular system with an implanted rotary assist device: simulation study with a refined computer model. Artificial Organs 26(4), 349-359.

Westerhof, G. Elzinga and P. Sipkema (1971). An artificial arterialsystem for pumping hearts. $J$ Appl Physiol 31, 776-781. 